\title{
Representaciones sociales de salud y adherencia farmacológica antihipertensiva en población pehuenche
}

\author{
PATRICIO OLIVA $^{(1)}$ y CARMEN NARVÁEZ ${ }^{(1)}$
}

\section{RESUMEN}

La hipertensión arterial es uno de los principales factores de riesgo de enfermedades cardiovasculares en los distintos grupos culturales del pais ${ }^{l}$. Objetivo: Describir las representaciones sociales pehuenches sobre el tema de la adherencia antihipertensiva farmacológica dentro de la lógica sanitaria cultural pehuenche de la comuna de Alto Bío Bío, Material y método: Es una investigación cualitativa, de carácter fenomenológico, donde se emplea un muestreo no probabilístico, de caso consecutivo en la población pehuenche, recolectando la información mediante entrevistas semiestructuradas. El análisis de los resultados es semántico estructural, lo cual permite describir pertinentemente las estructuras culturales sanitarias. Resultados: Éstos indican que el problema de la adherencia en pacientes indígenas radica en que los fármacos son vistos como "calmantes" que no curan las causas de las enfermedades, debido a que éstas, en un contexto étnico, se encuentran en un plano físico, espiritual y religioso, desde un punto de vista cultural, mágico y religioso, y no en contextos clínicos e individuales. Conclusión: los pehuenches poseen problemas de adherencia farmacológica antihipertensiva, siendo definida como el grado en que la conducta de un paciente, en relación con la toma de medicamentos coincide con las instrucciones proporcionadas por el médico ${ }^{2}$. Sus tradiciones no definen a la hipertensión como una enfermedad válida e histórica, en consecuencia la farmacoterapia resulta suntuaria y disociativa de ritos y tradiciones que mantienen viva su cultura. La farmacoterapia antihipertensiva es evaluada como un factor perjudicial a las instituciones tradicionales pehuenches.

Palabras clave: hipertensión, adherencia farmacológica, etnias.

\begin{abstract}
PEHUENCHE SOCIAL REPRESENTATIONS OF ARTERIAL HYPERTENSION: THE PROBLEM OF ADHERENCE TO MEDICATION

Arterial hypertension is one of the principal risk factors of cardiovascular diseases among many different cultural groups in the countryl. Objective: Describe Pehuenche social representations relating to adherence to antihypertensive medications, within the health belief system of the Pehuenche community in the municipality of Alto Bio Bio. Material and method: This is a qualitative investigation, based on phenomenology, with a non-probabilistic sample of consecutive cases taken from the Pehuenche population. Information was collected through semi-structured interviews. Structural semantics was used to analyze the results, which allowed pertinent description of the health belief structure. Results: Results indicate that the problem of adherence to medications in indigenous patients lies in the belief that these medications are only "soothing" ("calmantes") and don't cure the causes of disease. This is due to the fact that, from a cultural, magical and
\end{abstract}

(1) Facultad de Odontología. Universidad del Desarrollo. Barros Arana 1735 Concepción. Chile. patriciooliva@udd.cl. 
religious viewpoint, the causes of disease are believed to be found on a physical, spiritual and religious level, and not in the clinical or individual context. Conclusion: The problem of adherence to antihypertensive medications, defined as the grade in which the patient's behavior towards taking medications coincides with the instructions provided by the doctor ${ }^{2}$, is found in the Pehuenche population. Because their traditions don't include hypertension as a valid and historically recognized disease, the treatment is seen as a luxury and is disengaged from the rites and traditions the keep their culture alive. Antihypertensive treatment is considered harmful to traditional Pehuenche institutions.

Keywords: hypertension, adherence to medications, ethnicity.

\section{INTRODUCCIÓN}

La hipertensión arterial es una de las enfermedades crónicas más prevalentes, ya que afecta al $21 \%$ de la población ${ }^{3}$, y sólo $33 \%$ de los pacientes hipertensos logra normalizar su presión arterial. El problema radica en que la mitad de estos pacientes en control abandona la atención en el primer año del diagnóstico ${ }^{4}$, de los que siguen bajo supervisión médica, sólo $50 \%$ toma al menos $80 \%$ de sus medicamentos ${ }^{5}$. Los elementos socioculturales que pueden explicar la baja adherencia al tratamiento antihipertensivo son $\left.^{6}: 1\right)$ Baja participación social 2) Red social inadecuada 3) Discapacidad física mayor y 4) Nivel de educación. Sin embargo, se han identificado más de 200 variables independientes que explican el fenómeno ${ }^{7}$. Esto se acentúa en ciertos grupos étnicos como los pehuenches mapuches, que con respecto a la HTA, los datos arrojan un $20,4 \%$ en hombres y $18,8 \%$ en mujeres. En la población pehuenche, estudios oficiales demuestran problemas de adherencia a tratamientos antihipertensivo, a pesar de las altas tasas de dicha enfermedad ${ }^{8}$.

Para comprender la problemática de la no adherencia se realiza un estudio de carácter cualitativo, fenomenológico, que busca conocer los elementos socioculturales que influyen en los individuos al momento de no continuar con un tratamiento farmacológico en una enfermedad asintomática como lo es la hipertensión arterial, en la comuna de Alto Bío Bío, donde el 100\% de la población es pehuenche, y su medicina tradicional tiene aún una fuerte penetración cultural.
En la investigación se evalúa progresivamente la percepción del problema de la adherencia farmacológica en la hipertensión arterial, comprendiendo su lógica epidemiológica, y cómo a partir de ella se construyen conceptos sanitarios que intervienen activamente en las conductas adherentes de los hipertensos pehuenches, evaluando siempre desde una perspectiva de identificación de conceptos y dimensiones culturales, propios de una investigación de carácter cualitativo.

\section{MATERIAL Y MÉTODO}

El universo son todos los pacientes hipertensos que asistieron por ese problema a los Cesfam de la comuna de Ralco. Los criterios de inclusión para la investigación son: individuos mayores de 18 años, que se atiendan en los Cesfam seleccionados; con Hipertensión Arterial Diagnosticada; con prescripción de tratamiento farmacológico, que estén en condiciones cognitivas y físicas como para responder, dado que el estudio es cualitativo, y en concordancia con el problema, no se investigó población adherente. El diseño muestral, para la presente investigación es de Caso-Tipo' no Probabilístico: debido al carácter cualitativo de la investigación, no existe cálculo muestral asociado. Por otro lado, la técnica de muestreo es intencional debido a que los individuos serán seleccionados de manera intencional de acuerdo a los criterios de selección correspondientes. Se entrevistó a todos los individuos que cumplían con estos criterios hasta cumplir con el punto de saturación de la información.

El diseño de investigación es cualitativo - 
fenomenológico, se estructura a partir del contenido y de la interpretación de la realidad a través del significado subjetivo ${ }^{10}$. Se escoge este tipo de diseño por la flexibilidad que presenta al momento de integrar elementos subjetivos concernientes a la adherencia terapéutica antihipertensiva, específicamente la farmacológica, en un grupo cultural delimitado geográfica y cognoscitivamente. La técnica de recolección de la información es la entrevista semiestructurada y grupos de discusión, a todos los grupos muestrales seleccionados en la comuna de Ralco, hipertensos que estén o estuvieron en tratamiento antihipertensivo farmacológico, hasta llegar al límite de saturación.

La estrategia de análisis de la información es el análisis semántico estructural. El análisis semántico estructural consta de una serie de códigos (de base y calificativos) que va develando información latente en el discurso de los entrevistados y que cobra sentido en la medida en que se relaciona con otros códigos, permite no sólo la construcción de interpretaciones textuales, sino que también de categorías que poseen un nivel de abstracción mayor, generando conceptos globales sobre la temática tratada ${ }^{11}$.

\section{RESULTADO}

La salud pehuenche es definida como armonía del individuo con los demás individuos y con su entorno, siendo la ruptura, causa de enfermedad $^{8}$, por lo cual, los principales problemas sanitarios quedan definidos dentro de ámbitos conceptuales-culturales. Lo que en este estudio se hace es analizar el problema de la adherencia farmacológica en la hipertensión arterial, a partir del análisis de los discursos de los individuos, de los cuales es posible establecer los siguientes puntos:

A) La relación asociativa entre las variables conceptuales sanitarias endoculturales y los factores asociados al cumplimiento médico étnico, se establece dentro de criterios históricos, religiosos y empíricos.

B) Los individuos de la etnia pehuenche validan sus sistemas sanitarios, y la interacción de los roles asociados a estos, principalmente machis y hierbateras. La credibilidad asociada a estos actores por los pacientes permite, en último término, aceptar o no las explicaciones acerca de las causas de sus enfermedades, y la adherencia a los tratamientos farmacológicos occidentales $^{12}$.

La medicina pehuenche se fundamenta en ritos en las cuales interviene la comunidad, siendo la enfermedad un elemento de evaluación social en relación con el vínculo existente entre el individuo, la divinidad, el entorno y la comunidad. En consecuencia las medicinas son construcciones culturales que responden a necesidades de un entorno social específico y en los cuales es posible distinguir una dimensión conceptual y otra conductual ${ }^{12}$.

Los resultados, dentro de estas dimensiones, indican lo siguiente:

a) La adherencia a tratamientos médicos occidentales por parte de los pehuenches depende del grado de integración con su cultura, mientras más integrado sea, más difícil será la adherencia a tratamientos. Ejemplo: "...vienen acá los doctores, están unos meses y uno ni los conoce... cómo le va a creer sus tratamientos si no lo conozco... él tampoco sabe qué es ser pehuenche..."(entrevistado $\left.\mathrm{N}^{\circ} 4\right)$.

b) Las enfermedades crónicas no transmisibles, entre las cuales está la hipertensión arterial, no están presentes en los conceptos tradicionales históricos, lo que dificulta la incorporación de tratamientos a enfermedades que no son reconocidas. Ejemplo: "...los huincas vienen con sus médicos y explican enfermedades que nunca han existido... los antepasados nunca se habían enfermado de esto... porque no existe para el pehuenche..." (entrevistado $\mathrm{N}^{\circ} 2$ ).

c) Los tratamientos farmacológicos son contrapuestos con los tradicionales pehuenches basados en el uso de hierbas, determinando que: la enfermedad es producto de la ingesta de los medicamentos, la enfermedad es agravada por la ingesta de los medicamentos, la enfermedad es un efecto colateral de los medicamentos. Ejemplo: "... los remedios no son sanos... no 
vienen de la naturaleza... dañan al cuerpo porque no son naturales... no es como las hierbas... que tienen sabor y son ricas..." (entrevistado $\mathrm{N}^{\circ} 7$ ).

Por lo cual, de acuerdo al análisis discursivo, el tratamiento antihipertensivo es:

a) Innecesario, debido a que la hipertensión arterial culturalmente no existe. Ejemplo: "en nuestra historia no existen las mismas enfermedades que tienen los huincas... porque la alimentación es más sana... (entrevistado $\mathrm{N}^{\circ}$ ).

b) No responde a las tradiciones pehuenches, debido a que no existe un proceso de sanación que incorpore un procedimiento válido de recuperación dentro de la comunidad, lo que en el discurso se ejemplica como: "...los remedios nunca estuvieron con nuestros abuelos, además como que rompen con la ayuda que se le prestaba a los vecinos... no se sabe cuándo están enfermos... nadie participa ni hace rogativos ahora..." (entrevistado $\left.\mathrm{N}^{\circ} 12\right)$.

c) No responde a ciclos naturales presentes en la cultura pehuenche, debido a que el fármaco no se asocia a conceptualizaciones históricas, un ejemplo es: "... los remedios no son de la naturaleza... no tienen nada que ver con la tierra, y eso es malo..." (entrevistado $\mathrm{N}^{\circ} 4$ ).

d) Atenta contra los principios culturales pehuenches debido a que la efectividad del fármaco hace prescindir el proceso de sanación tradicional, que es más largo, involucra a la comunidad, y necesita de sanadores tradicionales.

En resumen, los pehuenches no adhieren porque sus tradiciones médicas les impiden hacerlo. Los resultados demuestran que el problema de la adherencia radica en que la incorporación de la farmacología en el sistema médico pehuenche altera el mantenimiento del rol de sanador dentro de la comunidad, el mantenimiento de los rituales y ceremonias que involucren el proceso de sanación del individuo, el carácter aleccionador de la enfermedad, la relación del individuo con el entorno y con su medio social y religioso.

\section{CONCLUSIÓN}

El territorio de Alto Bío Bío presenta una situación intercultural que deriva en la resistencia por parte de los pehuenches a incorporar la farmacología como parte de un tratamiento para dentro de enfermedades que no han sido catalogadas ni clasificadas culturalmente, manteniendo incólume sus conceptos sanitarios traiciónales, como ya se esbozaba en la evidencia cuantitativa de estudios previos ${ }^{8}$.

Los pehuenches de esa zona se enfrentan a la dicotomía de utilizar hierbas para sus enfermedades, como parte de expresiones culturales tradicionales, con un importante sustrato mágico-religioso. A esto se le incorpora el tratamiento farmacológico, en enfermedades que no han sido catalogadas ni clasificadas dentro de su lógica epidemiológica a lo largo de la historia, como lo son las enfermedades crónicas no transmisibles, específicamente la hipertensión arterial, para la cual, en su cultura no existe tratamiento. A pesar de que la cultura se define como una entidad dinámica ${ }^{13}$, se mantiene estático el concepto sanitario tradicional.

A diferencia de estudios anteriores ${ }^{14}$, en la presente investigación se demuestra como la intersubjetividades de los sujetos estudiados permiten generar construcciones sociales sanitarias sobre las enfermedades que no admiten variación temporal ni cultural. Esto se evidencia en la renuencia a adherir a tratamientos farmacológicos antihipertensivos que les resultan ajenos y contrarios a los conceptos culturales sanitarios que les fueron enseñados. Aún más, existe una negación del pehuenche a abandonar sus conceptos históricos sanitarios, justificando en distintos planos (individual, social y religioso, como se evaluó en los resultados) su elección por medicinas en base a hierbas, en contraposición a la farmacología considerada como ajena. Este enfrentamiento cultural se transforma en un problema de salud pública al ser un elemento decisivo en el cumplimiento de las metas sanitarias del país en dichas comunidades. 


\section{REFERENCIAS}

1. MINISTERIO DE SALUD. Encuesta Nacional de Salud Chile, 2003.

2. HAYNES RB. Introduction. En: Haynes RB, Taylor DW, Sackett DL, eds. Compliance in health care Baltimore: John Hopkins University Press; 1979. p. 1-7.

3. FASCE, E. estudio Tendencia en la Prevalencia, Conocimiento, Tratamiento y control de la Hipertensión Arterial en comunas urbanas de la VIII Región. Universidad de Concepción. Concepción. 2006.

4. MAPES RE. Physicians' drug innovation and relinquishment. Social Science \& Medicine. 1977. 11:619-624

5. SACKETT DL et al. Randomised clinical trial of strategies for improving medication compliance in primary hypertension.Lancet, 1975, 1:1205-1207

6. ROMAN A, OSCAR, VALENZUELA, MARÍA A, BADILLA S, MARTA et al. Optimización de la reducción de la presión arterial en hipertensos esenciales. Rev. Méd. Chile. Mayo 2002. vol 130, n5. p519 - 526. ISSN 0034-9887.

7. MORRELL RW, PARK DC, KIDDER DP Y MARTIN M (1997). Adherence to antihypertensive medications across the life span. The Gerontologist, 37, 609-619

8. Diagnóstico de la situación en salud de las comunidades pehuenches de Callaqui y Cauñicu. Apoyo al Diseño de Experiencias Piloto en Modelo de Salud Intercultural en las Comunidades Pehuenche de la Comuna de Alto Bío Bío, Octava Región. Provincia del Bío Bío. SS. Bío Bío. 2005.

9. HERNÁNDEZ, ROBERTO. Metodología de la investigación. México. Mc Graw Hill. 2003 p. 566.

10. SCHUTZ, ALFRED. El problema de la realidad social. Amorrotu editores. Argentina. 1995. P 115.

11. REMY, J. Métodos de análisis de contenido de Sociología. Bruselas, Publicación de Universidad Sain Louis. 1991:79.

12. ANA M ALARCÓN, ALDO VIDAL, JAIME NEIRA ROZAS. Salud intercultural: elementos para la construcción de sus bases conceptuales. Rev Méd Chile 2003; 131: 1064.

13. PARK, A. Introducing Anthropology. An Integrated Approach. Mayfield Publishing Company. California. 2000.

14. MORA, ZILEY. Estudio de Sistematización en Terreno de las Experiencias de Salud Intercultural en el Área de Desarrollo Indígena de Alto Bío Bío. impacto y presencia del sistema de salud en las comunidades indígenas pehuenches. Evaluación de programas, experiencias, dificultades, aciertos, catastros y propuestas sistémicas para un posible modelo de salud intercultural en Alto Bío Bío. Los Ángeles. Chile. Diciembre 2002.

Recepción: 29 abril 2009 Aprobación: 9 noviembre 2009

Usted puede comentar éste y otros artículos publicados en la Revista Chilena de Salud Pública, enviando un correo electrónico a revistasp@med.uchile.cl 